CERN-TH/96-214

hep-th/9608044

August 8th, 1996

\title{
Massive and Massless Supersymmetric Black Holes,
}

Contribution to the Proceedings of the Trieste Spring Workshop on String

Theory, Gauge Theory and Quantum Gravity, held in the I.C.T.P., Trieste, (Italy), the 28th and 29th of March 1996.

\author{
Tomás Ortín 円 \\ C.E.R.N. Theory Division \\ CH-1211, Genève 23, Switzerland
}

\begin{abstract}
We review some recent work on the existence and classification of extreme black-hole-type solutions in $N=8$ supergravity. For the black holes considered (those that are also solutions of $N=4$ supergravity and of the Einstein-Maxwell-dilaton theory with coupling a) a complete classification is achieved: the only possible values of $a$ are $\sqrt{3}, 1,1 / \sqrt{3}, 0$. Up to $\mathrm{U}$ duality transformations there is only one solution for each of those values. The exception is $a=0$ for which an additional extreme but non-supersymmetric Reissner-Nordström black hole solution exists.

We also study the so-called massless black-hole solutions. We argue that they can be understood as composite objects. At least one of the components would have "negative mass". We also argue that these states, being annihilated by all the generators in the supersymmetry algebra, could also constitute alternative vacua of the supergravity theory.
\end{abstract}

CERN-TH/96-214

\footnotetext{
${ }^{1}$ E-mail address: Tomas. Ortin@cern.ch
} 


\section{MASSIVE SUPERSYMMETRIC BLACK HOLES}

\subsection{INTRODUCTION}

Some of the most interesting classical solutions of string theory are extreme black holes. Black holes (BHs) are the closest relatives of massive point particles in gravitational physics. The masses of extreme BHs are completely determined by their charges. They are the extreme limit of regular charged BHs with two horizons (an event horizon and a Cauchy horizon) that coincide in this limit. In some cases they are regular BHs themselves and in other cases they are not, but in all cases, they become singular beyond that limit.

Part of the interest in extreme BHs is based on the fact that in many cases the extreme limit coincides with the limit in which some supersymmetry is restored. Configurations with unbroken supersymmetry enjoy very special properties: stability, non-renormalization theorems etc. and the states of the quantum theory whose field they describe are usually referred to as BPS states.

There are, though, some unsatisfactory aspects in this correspondence between extreme and supersymmetric BHs. To start with, the same metric can correspond to different solutions of the same supergravity theory (or even different supergravity theories). These solutions, usually called embeddings do not always have the same number of unbroken supersymmetries. Perhaps the best-known example of this situation is the extreme string dilaton $\mathrm{BH}$ $(a=1)$ Ref. [1, 2, 3]. When the vector field present in the solution is identified (as in Ref. [3]) as a Yang-Mills vector field belonging to a matter supermultiplet, the corresponding embedding is not supersymmetric. However, if the vector field is identified (as in Ref. [4]) with a graviphoton (one of the six of $N=4$ supergravity) the embedding has two out of four supersymmetries unbroken. Since the metric of both embeddings is evidently the same, it is hard to understand why in one case there is unbroken supersymmetry and in the other case there is not.

Our aim is to clarify this problem and we will give a partial solution. To this end we will study the embeddings of dilaton BHs simultaneously in $N=4$ and $N=8$ supergravity (low-energy heterotic and type II superstring effective actions). We will find that all embeddings of the same dilaton $\mathrm{BH}$ metric in $N=8$ supergravity are related by $\mathrm{U}$ duality transformations (i.e. there is a unique embedding modulo $\mathrm{U}$ duality) and have the same number of unbroken supersymmetries. However, the $N=4$ embeddings do not have the same number of unbroken supersymmetries, the reason being 
that they are not always related by a $\mathrm{T}$ or $\mathrm{S}$ duality transformation: the missing $\mathrm{U}$ duality transformation is not in the theory anymore.

There is, though, an exception to this situation for the Reissner-Nordström BH: there is a class of embeddings in $N=8$ which have no unbroken supersymmetries at all. One could, then, imagine a higher supergravity theory in which all the embeddings of the Reissner-Nordström BH where equivalent. A possible scenario in which this theory has a twelve-dimensional origin has been proposed [5]. Similar theories have also been proposed on different grounds (and with different signatures) [6, 6].

Now let us outline the procedure followed. First we obtain the action and supersymmetry transformation rules of ten-dimensional type IIA supergravity by dimensional reduction from $N=1, d=11$ supergravity. Setting the NS-NS fields to zero one gets $N=1, d=10$ supergravity. Any solution of the latter is automatically a solution of the former and this framework allows us to analyze simultaneously the unbroken supersymmetries of the same metric embedded in both theories. We further dimensionally reduce to four dimensions, getting $N=4$ supergravity coupled to six vector multiplets.

Then, in four dimensions we study which dilaton $\mathrm{BH}$ metrics can be embedded in $N=4(+6 V)$ supergravity. For the cases in which this is possible ( $a=\sqrt{3}, 1,1 / \sqrt{3}, 0)$, we rewrite the solutions in ten-dimensional notation using our previous results and study their unbroken supersymmetries in ten dimensions.

In Section 1.2 we present the extreme dilaton $\mathrm{BH}$ solutions and set up the problem of their embedding in $N=4(+6 V)$ supergravity. In Section 1.3 we give the results and comment on them. In Section 1.4 we deal with the non-supersymmetric embedding of the Reissner-Nordström BH.

This first half of the talk is mostly based on the work in Refs. [8, 5].

\subsection{THE EMBEDDING OF DILATON BHS IN $N=8$ SUPERGRAVITY}

Dilaton BHs are BH-type solution of the following action:

$$
S^{(a)}=\frac{1}{2} \int d^{4} x \sqrt{|g|}\left[-R-2(\partial \varphi)^{2}+\frac{1}{2} e^{-2 a \varphi} F^{2}\right] .
$$

that depends on the real positive constant $a$ and will be referred to as " $a$ model". The scalar $\varphi$ is in general different from the string dilaton which we denote by $\phi$. The corresponding equations of motion are 


$$
\left.\begin{array}{rl}
G_{\alpha \beta}+2 T_{\alpha \beta}^{\varphi}-e^{-2 a \varphi} T_{\alpha \beta}^{A} & =0, \\
\nabla^{2} \varphi-\frac{a}{4} e^{-2 a \varphi} F^{2} & =0, \\
\nabla_{\mu}\left(e^{-2 a \varphi} F^{\mu \alpha}\right) & =0,
\end{array}\right\}
$$

where $T_{\alpha \beta}^{\varphi}$ and $T_{\alpha \beta}^{A}$ are the energy-momentum tensors of the scalar field $\varphi$ and the vector field $A_{\mu}$ respectively and $G_{\alpha \beta}$ is the Einstein tensor. These equations admit extreme $\mathrm{BH}$ [9] and multi-BH solutions [10, 11] for all $a$. The purely electric extreme multi-BH solutions are

$$
\left\{\begin{aligned}
d s^{2} & =V^{-\frac{2}{1+a^{2}}} d t^{2}-V^{+\frac{2}{1+a^{2}}} d \vec{x}^{2} \\
e^{\varphi} & =V^{-\frac{a}{1+a^{2}}} \\
F_{t \underline{i}} & =-n \sqrt{\frac{2}{1+a^{2}}} \partial_{\underline{i}} V^{-1},
\end{aligned}\right.
$$

where $V(\vec{x})$ is a harmonic function in three-dimensional Euclidean space $\left(\partial_{\underline{i}} \partial_{\underline{i}} V=0\right)$ and $n= \pm 1$ gives the sign of the electric charges. The equations of motion of the $a$-model are invariant under the discrete electric-magnetic duality transformation

$$
F^{\prime}=e^{-2 a \varphi} F, \quad \varphi^{\prime}=-\varphi,
$$

and, therefore, a purely magnetic multi-BH solution always exists for any $a$. Furthermore, for the special values $a=0$ and $a=1$ dyonic solutions also exist [1, 2, 4], but we will need them here.

All these extreme solutions admit Killing spinors if one uses the appropriate definition of "gravitino" and "dilatino" supersymmetry transformation rules [12]. These rules coincide with the supersymmetry rules of known supergravity theories only in some cases, but it is not known exactly in which ones and for which values of $a$. It was argued in Ref. [13] that the cases $a=1 / \sqrt{3}, 1, \sqrt{3}, 0$, should appear in different consistent truncations of maximal $N=8$ supergravity.

Our first goal is to find for which values of $a$ the dilaton-BH solutions can be embedded in $N=4(+6 \mathrm{~V})$ supergravity (low-energy effective heterotic string theory or type IIA theory, in the sense explained before). This is the same as solving the following problem: given the equations of motion of $N=4(+6)$ supergravity

$$
G_{\alpha \beta}+2 T_{\alpha \beta}^{\phi}+\frac{9}{4} T_{\alpha \beta}^{B}
$$




$$
\begin{aligned}
-\frac{1}{4}\left[\partial_{\alpha} G_{m n} \partial_{\beta} G^{m n}-\frac{1}{2} g_{\alpha \beta} \partial_{\mu} G_{m n} \partial^{\mu} G^{m n}\right] & \\
-\frac{1}{4} G^{m n} G^{p q}\left[\partial_{\alpha} B_{m p} \partial_{\beta} B_{n q}-\frac{1}{2} g_{\alpha \beta} \partial_{\mu} B_{m p} \partial^{\mu} B_{n q}\right] & \\
+\frac{1}{2} G_{m n}\left[F^{(1) m}{ }_{\alpha}^{\mu} F^{(1) m}{ }_{\beta \mu}-\frac{1}{4} g_{\alpha \beta} F^{(1) m}{ }_{\mu \nu} F^{(1) m \mu \nu}\right] & \\
+\frac{1}{2} G^{m n}\left[\mathcal{F}_{m \alpha}{ }^{\mu} \mathcal{F}_{n \beta \mu}-\frac{1}{4} \tilde{g}_{\alpha \beta} \mathcal{F}_{m \mu \nu} \mathcal{F}_{n}{ }^{\mu \nu}\right] & =0, \\
\nabla^{2} \phi+\frac{3}{4} e^{-4 \phi} H^{2}+\frac{1}{8} e^{-2 \phi}\left[G_{m n} F^{(1) m} F^{(1) n}+G^{m n} \mathcal{F}_{m} \mathcal{F}_{n}\right] & =0, \\
\nabla^{2} G^{r s}-G^{m(r} G^{s) n} G^{p q}\left[\partial G_{m p} \partial G_{n q}+\partial_{m p} \partial B_{n q}\right] & \\
+\frac{1}{2} e^{-2 \phi}\left[F^{(1) r} F^{(1) s}-G^{m(r} G^{s) n} \mathcal{F}_{m} \mathcal{F}_{n}\right] & =0, \\
\left.\nabla_{\mu}\left(G^{n r} G^{q s} \partial^{\mu} B_{n q}\right)+e^{-2 \phi} \mathcal{F}_{m} G^{m[s} F^{(1) r}\right] & =0 \\
\nabla_{\mu}\left(e^{-2 \phi} G_{m n} F^{(1) n \mu \alpha}\right) & =0, \\
\nabla_{\mu}\left(e^{-2 \phi} G^{m n} \mathcal{F}_{n}{ }^{\mu \alpha}\right) & =0, \\
\nabla_{\mu}\left(e^{-4 \phi} H^{\mu \alpha \beta}\right) & =0,
\end{aligned}
$$

where $T_{\alpha \beta}^{\phi}$ and $T_{\alpha \beta}^{B}$ are the energy-momentum tensors of $\phi$ and $B_{\alpha \beta}$, in how many ways can they be reduced to those above of the $a$-model? One also has to take into account the Bianchi identities

$$
\begin{aligned}
\partial F^{(1) m} & =0, \quad \partial H=\frac{1}{2} F^{(1) m} F_{m}^{(2)}, \\
\partial F_{m}^{(2)} & =0 .
\end{aligned}
$$

It is clear that, to reduce these equations to the equations of the $a$-model, which only has one scalar and one vector field one has to identify the different vector field strengths either with $F$ or with ${ }^{\star} F$ or with linear combinations of both. Something analogous happens with the scalars. The result is that only in the cases $a=\sqrt{3}, 1,1 / \sqrt{3}, 0$ this can be done and it can be done in a few ways and under certain constraints: $F$ has to be purely electric (or magnetic). This is explained in the next section. 


\section{$1.3 \quad N=8$ SUPERSYMMETRIC BLACK HOLES}

Our results are collected in Table 11. When the $N=4(+6 \mathrm{~V})$ fields of the top row take the values given in the following rows, in terms of $\varphi$ and $F$, where $F$ is either purely electric or purely magnetic, then the $N=4(+6 \mathrm{~V})$ equations of motion reduce to those of the $a$-model for the value of $a$ given in the first column.

These are all the possible embeddings up to $\mathrm{S}$ or $\mathrm{T}$ duality symmetries of the $N=4(+6 \mathrm{~V})$ theory. Once the embeddings are known, it is easy to re-express the solutions as solutions of $N=1, d=10$ and $N=2 A, d=10$ supergravity and study the unbroken supersymmetries. The supersymmetries of the $N=1$ theory correspond to one chiral sector of the $N=2 \mathrm{~A}$ theory (conventionally, the positive chirality sector) and are given in last column under $n_{+}$. The unbroken supersymmetries of the other chiral sector, which are in the $N=2 A$ but not in the $N=1$ theory are under $n_{-}$. For each value of $a$ allowed, the total unbroken supersymmetry in the $N=2 \mathrm{~A}$ theory $\left(n_{+}+n_{-}\right)$is always the same, but $n_{ \pm}$are in general different for each $N=1$-inequivalent embedding, as explained in the introduction.

\subsection{THE EXCEPTION TO THE RULE}

The only exception corresponds to the last row in Table 1 which is a dyonic embedding of the Reissner-Nordström BH metric with no supersymmetries and, consequently, inequivalent under $\mathrm{U}$ duality to the other embeddings. The corresponding ten-dimensional configuration is purely gravitational

$$
\left\{\begin{aligned}
d \hat{s}^{2}= & V^{-2} d t^{2}-V^{2} d \vec{x}^{2}-\left[d x^{\underline{4}}+\sqrt{2} n\left(V^{-1} d t \pm V_{\underline{i}} d x^{\underline{i}}\right)\right]^{2} \\
& -d x^{\underline{I}} d x^{\underline{I}}, \quad I=5, \ldots, 9 . \\
\hat{B}= & \hat{\phi}=0,
\end{aligned}\right.
$$

where the $V_{\underline{i}}$ 's are functions satisfying

$$
\partial_{[\underline{i}} V_{j]}=\frac{1}{2} \epsilon_{i j k} \partial_{\underline{k}} V
$$

and this makes it very easy to check that $n_{+}=n_{-}=0$.

It is somewhat surprising to find that the solution to our problem (namely embedding the $N=4(+6 \mathrm{~V})$ solutions into maximal supergravity so all the

\footnotetext{
${ }^{2}$ An $N=1$ theory with negative chirality can also be constructed and fits in the negative chirality sector of the $N+2 A$ theory as explained in Ref. [8].
} 


\begin{tabular}{|c|c|c|c|c|c|c|c|c|}
\hline$a$ & $\phi$ & $\rho_{1}$ & $\rho_{2}$ & $F^{(1) 1}$ & $F^{(2)}{ }_{1}$ & $F^{(1) 2}$ & $F_{2}^{(2)}$ & $\left(n_{+}, n_{-}\right)$ \\
\hline$\sqrt{3}$ & $\frac{1}{\sqrt{3}} \varphi$ & $-\frac{2}{\sqrt{3}} \varphi$ & 0 & $\sqrt{2} F$ & 0 & 0 & 0 & $\left(\frac{1}{2}, \frac{1}{2}\right)$ \\
\hline \multirow[t]{3}{*}{1} & $\varphi$ & 0 & 0 & $F$ & $-F$ & 0 & 0 & $\left(\frac{1}{2}, 0\right)$ \\
\hline & $\varphi$ & 0 & 0 & $F$ & $+F$ & 0 & 0 & $\left(0, \frac{1}{2}\right)$ \\
\hline & 0 & $-\varphi$ & $\varphi$ & $F$ & 0 & $e^{-2 \varphi \star} F$ & 0 & $\left(\frac{1}{4}, \frac{1}{4}\right)$ \\
\hline \multirow[t]{2}{*}{$\frac{1}{\sqrt{3}}$} & $-\frac{1}{3} \varphi$ & $-\frac{2}{3} \varphi$ & 0 & $\sqrt{\frac{2}{3}} F$ & 0 & $\sqrt{\frac{2}{3}} e^{2 \phi \star} F$ & $-\sqrt{\frac{2}{3}} e^{2 \phi \star} F$ & $\left(\frac{1}{4}, 0\right)$ \\
\hline & $-\frac{1}{3} \varphi$ & $-\frac{2}{3} \varphi$ & 0 & $\sqrt{\frac{2}{3}} F$ & 0 & $\sqrt{\frac{2}{3}} e^{2 \phi \star} F$ & $+\sqrt{\frac{2}{3}} e^{2 \phi \star} F$ & $\left(0, \frac{1}{4}\right)$ \\
\hline \multirow[t]{3}{*}{0} & 0 & 0 & 0 & $\frac{1}{\sqrt{2}} F$ & $-\frac{1}{\sqrt{2}} F$ & $\frac{1}{\sqrt{2}}{ }^{\star} F$ & $-\frac{1}{\sqrt{2}}^{\star} F$ & $\left(\frac{1}{4}, 0\right)$ \\
\hline & 0 & 0 & 0 & $\frac{1}{\sqrt{2}} F$ & $+\frac{1}{\sqrt{2}} F$ & $\frac{1}{\sqrt{2}}^{\star} F$ & $+\frac{1}{\sqrt{2}}^{\star} F$ & $\left(0, \frac{1}{4}\right)$ \\
\hline & 0 & 0 & 0 & $F \pm{ }^{\star} F$ & 0 & 0 & 0 & $(0,0)$ \\
\hline
\end{tabular}

Table 1: Table of embeddings and supersymmetries of dilaton BHs. 
embeddings become equivalent) does not work in this case. A possibility is that there exists a higher supergravity theory that includes $N=8$ as a consistent truncation and which has a bigger duality group which makes absolutely all embeddings of the same metric equivalent. A possible scenario [5] is related to the presence of an $S L(2, \mathbb{Z})$ duality group in the $N=2 B$ supergravity theory whose origin could be twelve-dimensional [14].

\section{MASSLESS BLACK HOLES}

This second half of the talk is based on Ref. [15.

In General Relativity the ADM mass $m$ of an asymptotically flat space, which describes an isolated system, is the total energy of that system. Therefore, zero ADM mass should mean empty (i.e. flat) space. Indeed, when the ADM mass $m$ of the Schwarzschild metric goes to zero

$$
\left(1-\frac{2 m}{r}\right) d t^{2}-\left(1-\frac{2 m}{r}\right)^{-1} d r^{2}-r^{2} d \Omega^{2} \stackrel{m \rightarrow 0}{\longrightarrow} d t^{2}-d \vec{x}^{2}
$$

it approaches Minkowski's. However, things are different for the ReissnerNordström (RN) metric:

$$
\begin{aligned}
\left(1-\frac{2 m}{r}+\frac{q^{2}}{r^{2}}\right) d t^{2} & -\left(1-\frac{2 m}{r}+\frac{q^{2}}{r^{2}}\right)^{-1} d r^{2}-r^{2} d \Omega^{2} \\
& \stackrel{m \rightarrow 0}{\longrightarrow}\left(1+\frac{q^{2}}{r^{2}}\right) d t^{2}-\left(1+\frac{q^{2}}{r^{2}}\right)^{-1} d r^{2}-r^{2} d \Omega^{2}
\end{aligned}
$$

This metric describes a charged massless system and has a naked singularity at $r=0$. If we were considering macroscopic objects we could invoke Penrose's cosmic censorship hypothesis (which is an statement on the evolution of gravitating systems): the above metric will never be the result of the gravitational collapse of energetically well-behaved matter.

In fact, we know that the presence of an electromagnetic field gives a positive contribution to the total energy. There must be a negative contribution to compensate it which would already be present before gravitational collapse

\footnotetext{
${ }^{3}$ The singularity would be covered by a regular horizon if the sign of $q^{2}$ was reversed or the electric charge $q$ was Wick-rotated. This solution, describing a massless genuine black hole would not be a solution of the Einstein-Maxwell theory anymore, but it would be a solutions of the Einstein-anti-Maxwell theory considered by Gibbons and Rasheed in Ref. [16]. We will not consider this kind of theories here.
} 
took place and would violate the energy conditions of the cosmic censorship hypothesis. However, in GR it is impossible to give a rigorous definition of a local energy density and, if we are only given the metric describing the final state, we cannot say where the negative energy is and what its origin is.

The cosmic censorship hypothesis, though, does not apply to miscroscopic objects which are not the result of gravitational collapse. In fact, we do not know how bad the effect of the naked singularity of a microscopic object is, since, anyway, this metric would only be the (far field) low-energy effective description of that object.

Here is where supersymmetry comes to our rescue: the mass of all the quantum states of a supersymmetric theory satisfy a so-called Bogmol'nyi bound [17]. In particular, we could consider the RN metric as a solution of $N=2$ supergravity (which is just Einstein-Maxwell's theory when all the fermions are set to zero) describing the field of an associated quantum state of mass $m$ and charge $q$. The B-bound in this theory is simply

$$
m \geq|q+i p|,
$$

where $p$ is the magnetic charge. In these conditions we could say that the singular metric (16) does not describe the gravitational field of any state of the theory and we could discard it. Massless charged states of pure $N=2$ supergravity seem to be excluded and also supersymmetry seems to act as a cosmic censor at the microscopic level [4] (but see later).

For higher $N$ extended supergravities the B-bound depends on the moduli $\phi_{0}: m \geq\left|Z\left(\phi_{0}, q, p\right)\right|$ and it was argued in [18 that for specific values of the moduli, the form $Z\left(\phi_{0}, q, p\right)$ could be singular and become zero for certain values of the charges. States saturating the bound would be massless, and the appearance of these new massless states would be the signal of symmetry enhancement和.

Soon after this proposal was made, massless supersymmetric (i.e. Bbound-saturating) black holes were found in Refs. [19, 20, 21]. The canonical metric of the one we are going to concentrate in is

$$
d s^{2}=\left(1-\frac{D^{2}}{r^{2}}\right)^{-\frac{1}{2}} d t^{2}-\left(1-\frac{D^{2}}{r^{2}}\right)^{\frac{1}{2}} d \vec{x}^{2} .
$$

Objects of this kind cannot be excluded using our previous arguments:

\footnotetext{
${ }^{4}$ One has to be a bit careful in the interpretation of this result, though. The B-bound formula is deduced from the supersymmetry algebra assuming that one is dealing with a massive representation and then going to the rest frame $p^{\mu}=(m, \overrightarrow{0})$. The analysis is not valid anymore at the singular point in moduli space where the representation becomes massless. At the critical point, a different analysis is required for that representation.
} 
they are allowed by supersymmetry and they are also exact solutions of string theory. Understanding the nature of these objects is not only a challenge but a must to be able to exclude them or to rightly include them in the theory. Our purpose here will be to elaborate a model for them. We start by reviewing some of their main features:

1. The metric is singular when $r=|D|$. The singularity is a curvature singularity (observe that the area of spheres of radius $r, 4 \pi\left(1-\frac{D^{2}}{r^{2}}\right)^{\frac{1}{2}} r^{2}$, goes to zero in that limit).

2. This metric does not seem to be the extreme limit of any regular charged black-hole metric.

3. The expansion of the $g_{t t}$ component of the metric far away from the singularity, where the gravitational field is weak is

$$
g_{t t}=1+\frac{D^{2}}{2} \frac{1}{r^{2}}+\frac{3 D^{4}}{8} \frac{1}{r^{4}}+\mathcal{O}\left(\frac{1}{r^{6}}\right) .
$$

The coefficient of the $\frac{1}{r}$ term is, by definition, $-2 m$ and so the ADM mass of these objects is zero. In this limit, $g_{t t} \sim 1+2 \Phi$, where $\Phi$ is the Newtonian gravitational potential. Therefore,

$$
\Phi \sim \frac{D^{2}}{4} \frac{1}{r^{2}}+\frac{3 D^{4}}{16} \frac{1}{r^{4}}
$$

and has weakly repulsive character when acting on usual test particles [20].

4. They do not seem to move at the speed of light (or to move at all). In GR, an object moving at the speed of light would be represented by a gravitational wave. But this object is not a wave because it does not admit any light-like Killing vector to start with. On the other hand, usual objects with zero rest mass moving at the speed of light have positive total energy and non-zero three-momentum, unlike massless BHs. Actually, the whole ADM four-momentum of these objects vanishes.

5. When they are rightly embedded in a supergravity theory, they have half of $N=2$ or $N=4$ supersymmetries unbroken and the low-energy solutions describing them are also exact solutions of string theory. 
6. The last two properties imply stability: they cannot decay into anything else because they have the minimal mass allowed by supersymmetry (they saturate a B-bound) and the minimal mass allowed in Physics (zero).

7. There are no known metrics describing an arbitrary number of these objects in static equilibrium.

This last point deserves some further explanation. In the Einstein-Maxwell theory (which is the bosonic sector of $N=2$ supergravity) there is a family of solutions known as the Majumdar-Papapetrou (MP) solutions which are given in terms of a real function $V(\vec{x})$ harmonic in three-dimensional Euclidean space $\partial_{\underline{i}} \partial_{\underline{i}} V=0$

$$
\left\{\begin{aligned}
d s^{2} & =V^{-2} d t^{2}-V^{2} d \vec{x}^{2} \\
F_{t \underline{i}} & = \pm \sqrt{2} \partial_{\underline{i}} V^{-1}
\end{aligned}\right.
$$

$V$ can be chosen at will. All these solutions admit Killing spinors 22 and for choices that make the metric asymptotically flat, they are supersymmetric. The most interesting choice for $V$ is with point-like singularities:

$$
V(\vec{x})=1+\sum_{i} \frac{m_{i}}{\left|\vec{x}-\vec{x}_{i}\right|}
$$

When all the constants $m_{i}>0$, the metric describes a set of extreme RN black holes in static equilibrium [23] with charges $\pm m_{i}$. The (total) ADM mass is $m=\sum_{i} m_{i}$. One is tempted to say that the $i t h \mathrm{BH}$ has mass $m_{i}$, but there is no way to prove this, no matter how logical this conclusion seems to be, and the only well-defined mass in this system is $m$. However, thinking in terms of the individual masses can be physically helpful.

It is then possible to have supersymmetric solutions with zero ADM mass if we choose the constants $m_{i}$ (now positive and negative) such that $\sum_{i} m_{i}=$ 0 . For instance, we can have pairs of objects with opposite values of $m_{i}$. Each pair, if it was isolated, would be massless and we could say that the solution describes many of these massless pairs in equilibrium (even though the mass of each pair is not well defined). The constituents of these pairs would annihilate each other if placed at the same point and, therefore, this kind of solutions are quantum-mechanically unstable.

The only solutions known so far describing many massless BHs are of this kind. They never describe many objects of the type we are considering, but pairs of objects. This observation, together with the fact that elementary massless objects move at the speed of light, will prove important in what 
follows, because they are against an interpretation of massless BHs as elementary objects. Furthermore, the fact that there is a gravitational field although the total mass is zero suggests that the source of the gravitational field is an object with complex structure whose far field is approximately described by the above metric. It will be useful to establish an electrostatic analogy.

\subsection{AN ELECTROSTATIC MODEL}

Let us consider the electrostatic potential $\phi$ of a spatially confined charge distribution. If we perform a multi-pole expansion and find that the $1 / r$ term is absent, we would immediately conclude that the total charge is zero. However, if the field is not trivial, we must also conclude that there is some charge, in fact, as much positive as negative charge. This system resembles our massless $\mathrm{BH}$ but there is also a big difference: the field cannot be spherically symmetric (the only spherically symmetric term is the $1 / r$ one).

To get closer to the massless BH gravitational field one has to consider charge distributions that fill the whole space. This is not surprising because of the non-Abelian nature of the gravitational field: the gravitational field fills the whole space and is the source of gravitational field. Thus, let us consider two different, spherically symmetric, charge distributions $\rho^{(+)}(r)>0$ and $\rho^{(-)}(r)<0$ such that

$$
\int d V \rho^{(+)}=Q>0, \quad \int d V \rho^{(-)}=-Q .
$$

so the total charge density is $\rho(r)=\rho^{(+)}+\rho^{(-)} \neq 0$ is also spherically symmetric and the total charge is zero. At a distance $r$ of the origin, the electrostatic potential behaves as

$$
\phi(r) \sim Q(r) / r
$$

where $Q(r)$ is the charge contained in a sphere of radius $r$

$$
Q(r)=\int_{r^{\prime} \leq r} d V \rho\left(r^{\prime}\right)
$$

Now, if the charge densities are such that, for large $r, Q(r) \sim 1 / r$, then $\phi \sim 1 / r^{2}$, which is the large $r$ behavior of the Newtonian potential for the massless BHs.

Then, we have been able to generate a spherically symmetric electrostatic field with zero total charge but at the expense of having positive and negative charge densities in the whole space. 


\subsection{A MODEL FOR MASSLESS BHS}

The electrostatic analogy together with the previous discussion suggest a model for a massless $\mathrm{BH}$ : a massless $\mathrm{BH}$ would be a composite object, the components being two "energy distributions" that separately would correspond to equal but opposite total masses. (In GR a point-like object gives an energy distribution that fills the whole space.)

But this system is unstable classically (the components repel each other) and quantum-mechanically (they could annihilate each other). Furthermore, since the components repel each other, there would be a non-vanishing interaction energy and the total system would not be massless as we naively expected.

To solve these problems we need to have additional interactions so the total force felt by each component vanishes and the interaction energy also vanishes. This behavior is common when there is unbroken supersymmetry and this is essentially what we have in the massless MP solutions. We need, however, more than one additional kind of charge to overcome the possibility of annihilation between pairs with opposite amount of the same kind of charge that we found in the massless MP solutions. But, if the components carry different kinds of charge, they would not interact! This new problem is solved by the presence of scalar fields which interact with different kinds of charges.

Then, massless BHs of the type we are considering could be found in supergravity theories with $N>2$ (or $N=2$ with matter multiplets) and they could be made out of pairs with opposite "masses" and unbroken supersymmetry (in multi-BH solutions).

It is not difficult to check this hypothesis. The multi-BH solution we need was found in Refs. [24, 25] and later rediscovered in Ref. [26] in the framework of the theory described by the following simple action

$$
\begin{aligned}
S= & \int d x^{4} \sqrt{-g}\left\{-R-2\left[(\partial \phi)^{2}+(\partial \sigma)^{2}+(\partial \rho)^{2}\right]\right. \\
& +\frac{1}{4} e^{-2 \phi}\left[e^{-2(\sigma+\rho)}\left(F^{(1) 1}\right)^{2}+e^{-2(\sigma-\rho)}\left(F^{(1) 2}\right)^{2}\right. \\
& \left.\left.+e^{2(\sigma+\rho)}\left(F^{(2)} 1\right)^{2}+e^{2(\sigma-\rho)}\left(F^{(2)}\right)^{2}\right]\right\},
\end{aligned}
$$

which is a truncation of the low-energy effective action of the heterotic string [27] ( $N=4$ supergravity plus 22 vector multiplets).

The solution is given in terms of four independent harmonic functions $H^{(1)}, K^{(1)}, H^{(2)}, K^{(2)}\left(\partial_{\underline{i}} \partial_{\underline{i}} H=\partial_{\underline{i}} \partial_{\underline{i}} K=0, \quad i=1,2,3\right)$ 


$$
\begin{aligned}
d s^{2} & =U^{-\frac{1}{2}} d t^{2}-U^{\frac{1}{2}} d \vec{x}^{2}, \quad U=H^{(1)} K^{(1)} H^{(2)} K^{(2)} \\
e^{-4 \phi} & =\frac{H^{(1)} H^{(2)}}{K^{(1)} K^{(2)}}, \quad e^{-4 \sigma}=\frac{H^{(1)} K^{(2)}}{H^{(2)} K^{(1)}}, \quad e^{-4 \rho}=\frac{H^{(1)} K^{(1)}}{H^{(2)} K^{(2)}}, \\
F_{t \underline{i}}^{(a) 1} & =c^{(a)} \partial_{\underline{i}} \frac{1}{H^{(1)}}, \quad \tilde{F}^{(a) 2}{ }_{t \underline{i}}=d^{(a)} \partial_{\underline{i}} \frac{1}{K^{(1)}}, \quad a=1,2 .
\end{aligned}
$$

where $\left(c^{(a)}\right)^{2}=\left(d^{(a)}\right)^{2}=1$ and

$$
\tilde{F}^{(1) 2}=e^{-2(\phi+\sigma-\rho) \star} F^{(1) 2}, \quad \tilde{F}_{2}^{(2)}=e^{-2(\phi-\sigma+\rho) \star} F^{(2)}{ }_{2},
$$

and ${ }^{\star} F$ is the Hodge dual of $F$. Usually, the $H$ 's and $K$ 's are chosen to be strictly positive, that is, all the constants in

$$
H^{(a)}=1+\sum_{n} \frac{q_{n}^{(a)}}{\left|\vec{x}-\vec{x}_{n}\right|}, \quad K^{(a)}=1+\sum_{n} \frac{p_{n}^{(a)}}{\left|\vec{x}-\vec{x}_{n}\right|},
$$

are non-negative to avoid singularities in the metric, but we are after solutions with some negative $q$ 's or $p$ 's. Now, following Ref. [26], we consider solutions of the form

$$
H^{(a)}=1+\frac{q_{a}}{\left|\vec{x}-\vec{x}_{1}\right|}, \quad K^{(a)}=1+\frac{p_{a}}{\left|\vec{x}-\vec{x}_{2}\right|} .
$$

When all the $q$ 's and $p$ 's but one vanish, the solution is an $a=\sqrt{3}$ extreme dilaton $\mathrm{BH}$ if the non-vanishing constant is positive. Then, if several constants are positive, one can consider that the above solutions describes as many $a=\sqrt{3} \mathrm{BHs}$ in equilibrium. The ADM mass of the system is $m=\frac{1}{4}\left(q_{1}+p_{1}+q_{2}+p_{2}\right)$ and would be positive. When the coordinates of all the BHs coincide at one point (or we look to the system from far away so the distances between black holes are negligible) one gets $a=1,1 / \sqrt{3}, 0$ extreme dilaton $\mathrm{BH}$ which can be seen as a bound state with zero binding energy of elementary $a=\sqrt{3}$ BHs.

If we now take, for instance $q^{(1)}=-q^{2)}=D$ and $p^{(1)}=p^{(2)}=0$ (that is, an $a=\sqrt{3} \mathrm{BH}$ - "anti-BH" pairf, or dihole), we get the following massless metric

\footnotetext{
${ }^{5}$ The mass of each individual component would be $D / 4$ and $-D / 4$ if we could define the mass of each component, which is not possible: the only mass that can be rigorously defined is the total ADM mass of the whole space-time, $m$.
} 


$$
d s^{2}=\left(1+\frac{D}{r_{1}}\right)^{1 / 2}\left(1-\frac{D}{r_{2}}\right)^{1 / 2} d t^{2}-\left(1+\frac{D}{r_{1}}\right)^{-1 / 2}\left(1-\frac{D}{r_{2}}\right)^{-1 / 2} d \vec{x}^{2} .
$$

which reproduces the original massless $\mathrm{BH}$ metric (18) when $r_{1}=r_{2}=r$.

Following the same reasoning as in Ref. [26] we can conclude that the known massless BHs are the effective field of a bound state of a pair of objects with opposite masses, or dihole.

\subsection{CONCLUSION}

Massless black holes are objects with very strange properties. In particular, it is not clear what they are and whether they should be included in the theory. Their masslessness and the fact that they saturate at least a Bbound (because they are supersymmetric) mean together that they saturate all bounds (and "anti-bounds" of the form $m+\left|z_{i}\right| \geq 0$ ) and that they are in fact annihilated by all supersymmetry and Lorentz charges. In this respect they are analogous to Minkowski space which is also massless and is annihilated by all charges, being a vacuum state of the theory. Perhaps the answer to the question of whether massless black holes should move at the speed of light or not can be found on this analogy because nobody ever asks whether Minkowski's space should move at the speed of light. A vacuum state interpretation could perhaps be more appropriate for massless "black holes".

On the other hand, although we have not found any inconsistency in the inclusion of these admittedly rather exotic objects in the theory many more checks are clearly necessary. A check that has recently been performed in Ref. [28] is the study of the pair-production of these massless black holes. The study is based in a C-metric-type solution describing two massless black holes with opposite $U(1)$ charges accelerating apart. This happens without any additional external force. This is what would happen in QED between electrons and positrons if $e$ was imaginary, leading to vacuum instability under charged pair production. It is, therefore, not completely surprising that the action of related instanton describing the pair production of massless black holes indicates the corresponding instability of the vacuum. It is, perhaps, more surprising that two massless black holes with opposite charges seem to repel each other. The reason why this happens is not yet completely clear and should be investigated.

\section{References}


[1] G.W. Gibbons, Antigravitating Black Hole Solitons with Scalar Hair in $N=4$ Supergravity, Nucl. Phys. B207, (1982) 337.

[2] G.W. Gibbons and K. Maeda Black Holes and Membranes in Higher Dimensional Theories with Dilaton Fields, Nucl. Phys. B298, (1988) 741.

[3] D. Garfinkle, G. Horowitz and A. Strominger, Charged Black Holes in String Theory, Phys. Rev. D43 (1991) 3140, Erratum, ibid. D45 (1992) 3888.

[4] R. Kallosh, A. Linde, T. Ortín, A. Peet and A. Van Proeyen, Supersymmetry as a Cosmic Censor, Phys. Rev. D46 (1992) 5278-5302.

[5] R.R. Khuri and T. Ortín, A Non-Supersymmetric Dyonic Extreme Reissner-Nordström Black Hole, Phys. Lett. B373 (1996) 56-60.

[6] C.M. Hull, String Dynamics at Strong Coupling, Nucl. Phys. B468 (1996) 113-154.

[7] C. Vafa, Evidence for F Theory, Nucl. Phys. B469 (1996) 403-418.

[8] R.R. Khuri and T. Ortín, Supersymmetric Black Holes in $\mathrm{N}=8 \mathrm{Su}$ pergravity, Nucl. Phys. B467), (1996) 355-382.

[9] C.F. Holzhey and F. Wilczek, Black Holes as Elementary Particles, Nucl. Phys. B380 (1992) 447-477.

[10] K. Shiraishi, Multicentered Solution for Maximally Charged Dilaton Black Holes in Arbitrary Dimensions, J. Math. Phys. 34(4) (1993) 1480 .

[11] T. Ortín, Electric-Magnetic Duality and Supersymmetry in Stringy Black Holes, Phys. Rev. D47 (1993) 3136-3143.

[12] G.W. Gibbons, D. Kastor, L.A.J. London, P.K. Townsend and J. Traschen, Supersymmetric Self-gravitating Solitons, Nucl. Phys. B416 (1994) 850-880.

[13] C.M. Hull and P.K. Townsend, Unity of Superstring Dualities, Nucl. Phys. B438 (1995) 109-137.

[14] E. Bergshoeff, B. Janssen and T. Ortín, Solution-Generating Transformations and the String Effective Action, Class. Quantum Grav. 13 (1996) 321-343. 
[15] T. Ortín, Massless String Theory Black Holes as Black Diholes and Quadruholes, Phys. Rev. Lett. 76 (1996) 3890.

[16] G.W. Gibbons, and D.A. Rasheed, Dyson Pairs and Zero-Mass Black Holes, Cambridge U. Report DAMTP/96/22 and hep-th/9604177.

[17] E. Witten and D. Olive, Supersymmetry Algebras that Include Topological Charges, Phys. Lett. 78B (1978) 97.

[18] C.M. Hull and P.K. Townsend, Enhanced Gauge Symmetries in Superstring Theories, Nucl. Phys. B451 (1995) 525.

[19] K. Behrndt, About a Class of Exact String Backgrounds, Nucl. Phys. B455 (1995) 188-210.

[20] R. Kallosh and A. Linde, Exact Supersymmetric Massive and Massless White Holes Phys. Rev. D52 (1995) 7137-7145.

[21] R. Kallosh and A. Linde, Supersymmetric Balance of Forces and Condensation of BPS States, Phys. Rev. D53 (1996) 5734-5744.

[22] G.W. Gibbons and C.M. Hull, A Bogomolnyi bound for General Relativity and Solitons in $N=2$ Supergravity, Phys. Lett. 109B (1982) 190.

[23] J.B. Hartle and S.W. Hawking, Solutions of the Einstein-Maxwell Equations with Many Black Holes Commun. Math. Phys. 26, (1972) 87.

[24] M. Cvetič and D. Youm, Singular BPS Saturated States and Enhanced Symmetries of Four-Dimensional $N=4$ Supersymmetric String Vacua, Phys. Lett. B359 (1995) 87-92.

[25] M. Cvetič and A.A. Tseytlin, General Class of BPS Saturated Dyonic Black Holes as Exact Superstring Solutions Phys. Lett. B366 (1996) 95.

M. Cvetič and A.A. Tseytlin, Solitonic Strings and BPS Saturated Dyonic Black Holes, Phys. Rev. D53 (1996) 5619-5633.

[26] J. Rahmfeld, Extremal Black Holes as Bound States, Phys. Lett. B372 (1996) 198-203.

[27] J. Maharana and J.H. Schwarz, Non-Compact Symmetries in String Theory, Nucl. Phys. B390, (1993) 3-32. 
[28] R. Emparán, Massless Black-Hole Pairs in String Theory, University of California at Santa Barbara Report UCSBTH-96-17 and hep-th/9607102. 